\title{
Respuesta completa tras tratamiento con bioquimioterapia en un caso de melanoma cutáneo diseminado
}

\section{Complete response in a patient with a metastatic cutaneous melanoma}

\author{
M.J. Bermejo-Pérez, E. Villar-Chamorro, M. Lemos-Simosomo, \\ C. García-González, A.M. Galeote-Miguel
}

\section{RESUMEN}

Fundamento. El pronóstico del melanoma diseminado es muy sombrío. Actualmente no hay consenso sobre el tratamiento estándar en primera línea para el melanoma metastático. Se presenta un caso por su comportamiento excepcional.

Resultados. Varón de 43 años diagnosticado en 1999 de melanoma maligno estadio IIA. En mayo de 2000 se objetivaron metástasis hepáticas y esplénicas. Recibió 6 ciclos de bioquimioterapia (cisplatino y DTIC junto con interleukina-2 e interferón- $\alpha$ ) cada 21 días y otros 6 ciclos con inmunoterapia sola (interleukina-2 e interferón- $\alpha$ ). Actualmente el paciente sigue vivo y sin evidencia de enfermedad.

Conclusión. El melanoma cutáneo metastático, en ocasiones, presenta una inusual evolución favorable. Es de esperar que los métodos de detección de marcadores moleculares logren determinar factores implicados en este tipo de respuesta y que los nuevos tratamientos dirigidos consigan mantener esta tendencia positiva.

Palabras clave. Melanoma. Quimioterapia. Inmunoquimioterapia.

\begin{abstract}
Background. The management of patients with disseminated disease is a difficult problem. There is currently no consensus on the standard first-line treatment for metastatic melanoma. We present a case because of his exceptional evolution.
\end{abstract}

Results. A 43 year old male diagnosed in 1999 with malignant melanoma stage IIA. In May 2000, hepatic and splenic metastases were detected. He received 6 cycles of biochemotherapy (cisplatin and DTIC, plus interleukin-2 and interferon- $\alpha$ ) and another 6 cycles with single immunotherapy (interleukin-2 and interferon- $\alpha$ ). Today, the patient is still alive and without evidence of disease.

Conclusion. Metastatic cutaneous melanoma, sometimes, presents and unusual and favourable evolution. In the near future, the methods of detection of molecular markers are expected to identify factors involved in this type of response. Furthermore, new targeted therapies may become essential to maintain this positive trend.

Key words. Melanoma. Chemotherapy. Inmunotherapy.

An. Sist. Sanit. Navar. 2011; 34 (2): 307-310

Hospital Regional Carlos Haya. Málaga.

Recepción: 11 de febrero de 2011

Aceptación provisional: 7 de marzo de 2011

Aceptación definitiva: 21 de marzo de 2011

\author{
Correspondencia \\ María José Bermejo Pérez \\ Servicio Oncología Médica \\ Hospital Regional Carlos Haya \\ Avda. Carlos Haya, s/n \\ 29010 Málaga \\ E-mail: cheberpe@gmail.com
}




\section{INTRODUCCIÓN}

El pronóstico del melanoma diseminado es muy sombrío, estimándose la supervivencia a los 5 años en menos del $10 \%$, con una mediana en torno a los 12 meses tras el diagnóstico ${ }^{1}$. Actualmente no hay consenso sobre el tratamiento estándar en primera línea para el melanoma diseminado. Después de 40 años de investigación, la dacarbazina (DTIC) sigue siendo el agente quimioterápico más activo y el de elección para el melanoma metastático, a pesar de los pobres resultados que muestran los estudios aleatorizados: tasas de respuesta en monoterapia del $7,2-7,5 \%$ con supervivencia libre de progresión (SLP) de 2 meses y sin beneficios a largo plazo ${ }^{2}$. Se han ensayado numerosas modalidades de tratamiento para incrementar estos resultados, introduciendo nuevas drogas en combinación con DTIC o usando biomoduladores en diferentes pautas, generalmente asociados a la quimioterapia ${ }^{3}$. Con los nuevos esquemas de tratamiento se ha conseguido mejorar la tasa y duración de las respuestas, pero sin mejoría significativa de la supervivencia ${ }^{4}$. En definitiva, con los tratamientos actuales, la supervivencia de los pacientes diagnosticados de melanoma diseminado, sobre todo con metástasis viscerales, es muy corta. Se presenta un caso de comportamiento excepcional.

\section{CASO CLÍNICO}

Paciente varón de 43 años, que nota a principios de 1999 aumento de tamaño y sangrado sobre lesión dérmica pigmentada preexistente en región interescapular. En abril de ese año, tras la exéresis de dicha lesión, se diagnostica de melanoma maligno nivel II de Clark, con $3 \mathrm{~mm}$ de Breslow (Estadio IIA). Para obtener margen suficiente mayor de $1 \mathrm{~cm}$, en junio se procede a ampliar márgenes sin encontrar células neoplásicas en la pieza. La exploración física completa fue normal y el estudio de extensión con analítica general (incluía pruebas hepáticas, LDH, y perfil graso), radiografía simple de tórax, ecografía abdominal y gammagrafía ósea no mostraron ningún resultado patológico. Se recomendó seguimiento en consulta, autoexploración y evitar la exposición al sol.
En mayo del año 2000, se objetiva, en una ecografía de control, la presencia de tres lesiones ocupantes de espacio (LOES) en hígado (la mayor de 2,5 cm de diámetro máximo) junto a otras dos en bazo, sugestivas de metástasis. La biopsia percutánea dirigida por control ecográfico de una de estas lesiones hepáticas confirmó el diagnóstico de metástasis de melanoma. No se detectaron metástasis a otro nivel. En junio del año 2000 inicia tratamiento bioquimioterápico con cisplatino y DTIC intravenoso, combinado con la administración subcutánea de interleukina- 2 e interferón- $\alpha$ cada 21 días. Tras 6 ciclos de inducción el paciente recibe otros 6 ciclos de mantenimiento basados en inmunoterapia sola (interleukina-2 e interferón- $\alpha$ subcutáneo) con buena tolerancia, salvo leve toxicidad pseudogripal.

En agosto del 2000, el control ecográfico no detecta las lesiones esplénicas descritas previamente y en el TAC abdominal persiste solo la mayor de las LOES hepáticas, pero de un tamaño inferior $(1 \times 1 \mathrm{~cm})$.

Finaliza el tratamiento en febrero de 2001, fecha en que las pruebas radiológicas no evidencian enfermedad a ningún nivel y el paciente permanecía asintomático.

Continúa en vigilancia y en febrero de 2003 se realiza una tomografía por emisión de positrones (PET), que no muestra ninguna actividad sugestiva de metástasis. Se ha revisado cuidadosamente por varios patólogos la pieza histológica obtenida por punción-biopsia de una de las metástasis hepáticas, confirmando el diagnóstico inicial de melanoma. Es de destacar que, en mayo del 2005, presenta un episodio de artralgias generalizadas con radiografías selectivas y gammagrafía ósea normal. El cuadro cedió con antiinflamatorios en 3 meses.

A fecha de hoy, tras 10 años y 6 meses desde el diagnóstico, el paciente sigue vivo y sin evidencia de enfermedad.

\section{DISCUSIÓN}

Tanto la obtención de una remisión completa como de una larga supervivencia es extremadamente infrecuente en pacientes con melanoma diseminado, especialmente con afectación visceral ${ }^{1}$. De modo ocasional, otros autores han presentado casos similares, comunicándose hasta un 10\% de supervivencia a los 2 años, y cerca del 7\% a los 5 años. En estos casos se ha investigado sobre variables que podrían influir en 
una larga supervivencia ${ }^{5}$. Entre los factores favorables se encuentra la edad joven, el sexo femenino, la presencia de metástasis resecables -localizada en piel o ganglios linfáticos-, o metástasis única ${ }^{6}$. Además de los aspectos clínicos ya conocidos, diversos autores han insistido en la posible detección de factores determinantes del pronóstico, tanto en la pieza quirúrgica como en el suero, pero aún no existe un método preciso para predecir la respuesta en cada individuo $^{7,8}$. En el caso que nos ocupa no se daban factores clínicos favorables, no padecía ningún proceso patológico asociado ni tomaba ninguna otra medicación que pudiera justificar mecanismos de inducción de apoptosis o inhibición de neovascularización. Dada la previsible mala evolución del paciente en un plazo de tiempo breve, la primera medida fue confirmar el origen metastático de las lesiones hepáticas mediante la revisión de la pieza de biopsia, lo que se hizo al menos en dos ocasiones por dos patólogos distintos.

Publicaciones de estudios no controlados han mostrado respuestas prolongadas en algunos pacientes tratados con bioquimioterapia $^{9}$, como parece ser el caso. No obstante, estos resultados hay que analizarlos con cautela en ausencia de estudios que demuestren la superioridad de este esquema frente a otros tratamientos ${ }^{4,10}$.

Recientemente se ha publicado un ensayo fase III con resultados "esperanzadores» para los pacientes diagnosticados de melanoma metastáticos. La mediana de supervivencia fue de 10 meses para los tratados con ipilimumab y 6 para los no tratados, con una supervivencia a dos años del $24 \%$ en el brazo del ipilimumab, que se mantenían a lo largo del seguimiento ${ }^{11}$. Otros fármacos con capacidad selectiva de inhibición de la forma mutada de B-RAF (GSK218436/PLX4032) $^{12,13}$, de inhibición de la actividad tirosín-Kinasa de c-KIT (imatinib/sorafenib) ${ }^{14}$, así como de inducción de apoptosis a través de la vía intrínseca (tasisulam) ${ }^{15}$ también están mostrando resultados positivos. En el caso presentado no se pudieron determinar marcadores experimentales al no estar previsto en el estudio.
Podemos concluir que el melanoma maligno cutáneo, en ocasiones, presenta una inusual evolución favorable. Es de esperar que los nuevos métodos de detección de marcadores moleculares logren determinar factores implicados en este tipo de respuesta y que los nuevos tratamientos dirigidos consigan mantener esta tendencia positiva.

\section{Agradecimientos}

Nuestro agradecimiento al Dr. Bretón García, por su ayuda, su disponibilidad y su interés constante en nuestra formación.

\section{BIBLIOGRAFÍA}

1. Gershenwald JE, Morton DL, Thompson JF, Kirkwood JM, Soong S, BALChet CM et al. Collaborators of the AJCC/UICC Melanoma Task Force. Staging and prognostic factors for stage IV melanoma: initial results of an American Joint Committee on Cancer (AJCC) international evidence-based assessment of 4.895 melanoma patients. J Clin Oncol 2008; 26 (Supl): abstract 9035.

2. EgGermont AM, KirKwood JM. Re-evaluating the role of dacarbazine in metastatic melanoma: what have we learned in 30 years? Eur J Cancer 2004; 40: 1825-1836.

3. Eton O, Legha SS, BediKian AY, Lee JJ, BuZAid AC, HodGEs C et al. Sequential biochemotherapy versus chemotherapy for metastatic melanoma: results from a phase III randomized trial. J Clin Oncol 2002; 20: 2045-2052.

4. Ives NJ, Stowe RL, Lorigan P, Wheatley K. Chemotherapy compared with biochemotherapy for the treatment of metastatic melanoma: a meta-analysis of 18 trials involving 2.621 patients. J Clin Oncol 2007; 25: 54265434.

5. Brand CU, Ellwanger U, Stroebel W, Meier F, Schlagenhauff B, Rassner G et al. Prolonged survival of 2 years or longer for patients with disseminated melanoma. An analysis of related prognostic factors. Cancer 1997; 79: 2345-1353.

6. Melanoma of the skin. En: Edge SB, Byrd DR, Compton CC et al, editores. AJCC Cancer Staging Manual. 7th ed. New York, NY: Springer, 2010: 325-344. 
7. Potti A, Moazzam N, Langness E, Sholes K, TenDULKAR K, KoCH M et al. Immunohistochemical determinación of HER-2/neu, c-Kit (CD117), and vascular endothelial growth factor (VEGF) overexpression in malignant melanoma. J Cancer Res Clin Oncol 2004; 130: 80-86.

8. Meiriño R, Martínez E, Marcos M, Villafranca E, Domínguez MA, IllarRamendi JJ et al. Factores pronósticos en el melanoma maligno cutáneo. An Sist Sanit Navar 2001; 24 (Supl. 1): 167-172.

9. Minor DR, Moore D, Kim C, Kashani-Sabet M, Venna SS, Wang W et al. Prognostic factors in metastatic melanoma patients treated with biochemotherapy and maintenance immunotherapy. Oncologist 2009; 14: 995-1002.

10. Vega EF, Mayordomo Ji, Meana JA, Valladares M, Florián J, Bretón JJ et al. Bioquimioterapia ambulatoria con cisplatino, dacarbacina, inteleucina-2 e interferón alfa en pacientes con melanoma avanzado. Estudio multicéntrico en 44 pacientes. Rev Oncol 2003; 5: 79-87.

11. Hodi FS, O'day SJ, MCDermott DF, Weber RW, Sosman JA, HaAnEn JB et al. Improved survi- val with ipilimumab in patients with metastatic melanoma. N Engl J Med 2010; 363: 711723.

12. Flaherty KT, Puzanov I, Kim KB, Ribas A, McarTHUR GA, Sosman JA et al. Inhibition of mutated, activated BRAF in metastatic melanoma. N Engl J Med 2010; 363: 809-819.

13. Kefford R, Arkenau H, Brown PM. Phase I/II study of GSK218436, a selective inhibitor of oncogenic mutante BRAF kinase, in patients with metastatic melanoma and other solid tumors. J Clin Oncol 2010; 28 (Supl): 611s (abstract 8503).

14. Handolias D, Hamilton AL, Salemi R, Tan A, MooDIE K, KERR L et al. Clinical responses observed with imatinib or sorafenib in melanoma patients expressing mutations in KIT. Br J Cancer 2010; 102: 1219-1223.

15. Kirkwood JM, Gonzalez R, Reintgen DS, Clingan PR, Mcwilliams RR, Ilaria RL et al. A phase II study of tasisulam sodium (LY573636) as second-line treatment for patients with unresectable or metastatic melanoma. J Clin Oncol 2010; 28 (Supl 15): abstract 8541. 